\title{
Modeling and Analysis of HAPS-based Cellular IoT Systems for Protected Areas
}

\author{
Masoud Ardiny, Mahbub Hassan, Senior, IEEE, Ming Ding, Senior, IEEE, Omar Salim, Member, \\ IEEE, and Nemai Karmakar, Senior, IEEE
}

\begin{abstract}
Floating in the stratosphere at an altitude of $20 \mathrm{~km}$, a High Altitude Platform Station (HAPS) can provide cellular-like coverage to vast areas on earth. In this paper we consider such HAPS-based cellular systems to provide IoT coverage for protected areas, i.e., national parks, wilderness, natural reserves, rain forests, etc., where terrestrial cellular or other network coverage does not exist. We derive analytical models to study the relationships among various parameters of a HAPS-based cellular system. Our model allows us to maximize the coverage area under various constraints such as device density on the ground, battery life of the IoT sensors, and the size of onboard antennas for the HAPS. Through numerical experiments, we show that HAPSbased cellular systems are capable of delivering existing cellular IoT solutions, such as NB-IoT and LTE-m, with acceptable quality of service.
\end{abstract}

Index Terms - Cellular IoT, high altitude platform station (HAPS), LTE-m , NB-IoT, protected areas.

\section{INTRODUCTION}

$\mathrm{P}$ ROTECTED areas (PAs) refer to regions, such as national parks, wilderness, rainforests, etc., that are reserved for conserving biodiversity and ecosystem. According to the latest statistics, land PAs comprise $20,359,155 \mathrm{~km}^{2}$, which is equivalent to $15.1 \%$ of the earth's land[1]. Given that smart conservation of PAs demands big data, we require massive scale internet of things (IoT) deployments to gather reliable information from living and non-living things in these PAs. Required communication paradigms of such PA IoTs are as follows:

- Periodical Reports: The deployed IoT sensors send short 20-200-byte messages at specified intervals that can range from a few minutes to few hours. Delay-tolerant applications such as toxic leakage detection, water quality monitoring, etc., can make use of this service. We can also use smart ear tags and collars to monitor animal habitat in PAs.

- Alerting Reports: The time interval between alerting messages is unpredictable and for some applications the

This paragraph of the first footnote will contain the date on which you submitted your paper for review. It will also contain support information, including sponsor and financial support acknowledgment. For example, "This work was supported in part by the U.S. Department of Commerce under Grant BS123456."

Masoud Ardiny is with the department of Engineering, Shahed University,Tehran, Iran (e-mail: masoud.ardiny@gmail.com). volume of traffic can be significant. For example, in poaching and illegal logging detection, array acoustic sensors would send time-stamped raw data to the server until the exact location and nature of the hazard can be pinpointed. Alerting reports can also be a useful IoT tool for detecting unpredictable animal events such as when aquatic mammals come to the water's surface to breathe.

- Command Mode: Sending commands to unmanned robotic projects or simple actuators is one of the very useful applications for monitoring PAs. This mode could also be useful for sending text messages to rangers, tourists, and indigenous people.

The aforementioned PA IoT applications would require communication systems that must fulfill two important criteria: (1) the IoT devices must operate with very low-power so they last for many years with a small battery, and (2) the IoT network must be able to decode weak signals coming from IoT sensors that may be located far from a base station or in significant nonline-of-sight scenario, such as under deep foliage, mud, or water.

There are several terrestrial IoT solutions launched in recent years that are technically capable of meeting the demands of PA IoT. These are called low-power wide area network (LPWAN); they are available both in unlicensed band, such as LoRa[2], as well as in the licensed cellular bands such as NB-IoT and LTE$\mathrm{m}$, which are referred to as Cellular IoT[3]. Unfortunately, they are not practical for PA IoT as it is challenging and not advisable to deploy terrestrial communication infrastructure in preserved landscapes.

Aerial solutions are most appropriate for protected areas. Satellite communication is good for coverage, but not suitable for tiny battery-powered IoT sensors due to the extremely long distance $(36,000 \mathrm{~km}$ above ground for geostationary orbit (GEO) and almost $800 \mathrm{~km}$ for low earth orbit (LEO)) involved in such communications. Not only, the GEO satellites are expensive, but also their ground modems should be equipped with more powerful transmitters. On the other hand, the LEO satellites need a constellation. Aerial cellular services using low

Mahbub Hassan is with the School of Computer Science and Engineering University of New South Wales Sydney, NSW, Australia. (e-mail: mahbub.hassan@unsw.edu.au).

Ming Ding is with the CSIRO,Data61, Australia. (e-mail: Ming.Ding@data61.csiro.au)

Omar Salim and Nemai Karmakar are with the Department of Electrical and Computer Systems Engineering Monash University Melbourne, VIC, Australia (e-mail: omar.salim@monash.edu \& nemai.karmakar@monash.edu). 
altitude platforms, such as drones that fly very close to earth (less than a few hundred meters) [4], [5] is another possibility, but LAPs face major problems such as short battery life and flight time, collision hazard, security, privacy, regulation, and so on [6].

HAPS is more suitable to deploy PA IoT networks because they fly low enough ( $20 \mathrm{~km}$ from the earth surface) to enable low-power communications for ground sensors, and at the same time they are high enough where they can be potentially powered for months by solar energy[7], [8]. Also, compared to LAPs, a single HAP can cover a large area, which is really attractive for PAs. Indeed, in recent years, there have been a growing research interest in HAPS-based cellular systems that can connect existing cellular user equipment on the ground without any modifications [9].

Our goal in this paper is to analyze such HAPS-based cellular systems that can deliver the recently introduced NB-IoT and LTE-m services to IoT devices located in PAs. To realize this goal, we derive analytical models to study the relationships among various parameters of a HAPS-based cellular system considering the propagation models appropriate for PAs. Our model allows us to maximize the coverage area under various constraints such as device density on the ground, battery life of the IoT sensors, and the size of onboard antennas for the HAPS. Through numerical experiments, we show that it is practical to design HAPS-based cellular systems that are capable of delivering existing cellular IoT solutions, such as NB-IoT and LTE-m, to PAs with acceptable quality of service.

The rest of the paper is organized as follows. Related work is reviewed in Section II. We describe the system model in Section III followed by the numerical results in Section IV. Finally, Section V concludes this article and summarizes its key findings.

\section{RELATED WORK}

Although the abilities of IoT were proven for smart cities and other industrial environments, there is only little attempt to apply it for protected areas. For example, Yao et al. [10] have proposed a forward-wait-deliver strategy for long-term wildlife tracking and monitoring. The authors in [2] suggested LoRa Alliance and Bluetooth low energy (BLE) for the wildlife monitoring systems. In [11], the authors studied flexible and stretchable skin-like waterproof tags for marine species that can be connected using BLE. Olasupo [12] reports performance $\mathrm{XBee}$ and LoRa in rocky and mountainous environments. In [13], the authors have stated that WiFi-based long-distance networks (IEEE 802.11) and low-rate wireless personal area networks (IEEE 802.15.4) can be used to connect rural areas for agricultural applications (PAs can use this idea too). Most of the studies have established networks that use license-free protocol (LoRa, SigFox, etc.) thanks to the lack of cellular networks in protected areas.

The 3GPP has developed narrowband internet of things (NBIoT) and long-term evolution-m (LTE-m), often referred to as Cellular IoT, that can meet the requirements of machine-type communications [14]. NB-IoT and LTE-m modules can be implemented as low-cost system on chip (SoC), which can be easily integrated in a smart collar for monitoring endangered animals or in as smart tags for monitoring forests. The estimated battery life proves that these NB-IoT and LTE-m tags can work for a long time without needing battery replacement.

Some researchers investigated aerial wireless services using LAPs, such as drone-mounted flying base stations. For example, The authors in [15] suggested UAV-IoT networks for early wildfire detection. They have stated that wildfire detection at early stages is not possible using this satellite remote sensing method. However, large number of such drones would be required to cover PAs. Some study proposed constant movement of the drones for reducing the total number of drones [16], but when the alerting report wants to send a burst of data in a fraction of a second, the IoT device cannot wait for the drones to move and come close to it.

A HAP can cover a wide area and due to operating at $20 \mathrm{~km}$ in the stratosphere, where wind intensity and air turbulence are minimal and are immune from the storm and most natural disasters as well as are safe against subversive activities. So, in this paper, a HAP with multiple antennas is used instead of multiple LAPs with single antenna.

The authors in [17][18] have discussed cell configuration optimization for a HAPS cellular system, where the authors investigated different cell numbers like 7, 9, 12, 19, and 21 and derived the best configuration for each one. They focused on parameters like spectral efficiency and energy efficiency. Our study focuses on a new aspect of the problem: how to maximize the coverage under the practical constraints like the total size of the onboard antenna, the density of the IoT devices on the ground, and the uniformity of the quality of service across all the cells within the coverage area.

\section{SYSTEM MODEL}

Fig. 1 shows the HAPS cellular network topology to cover a protected area. Using a system of onboard antennas, a HAPS platform divides its coverage area into cells so that the available carriers can be reused in every cell to support high IoT device density (DD) expected in protected area monitoring applications. We consider a cell-specific antenna system, i.e., a separate antenna for each cell. Compared to massive MIMO antennas often considered in terrestrial base stations for creating sharp beams, the cell-specific antennas are expected to be more resilient to small vibrations of the HAPS platform. Also, the ground devices connect directly to the HAPS platform, which in turn uses either satellite or terrestrial backhaul to connect to the core network.

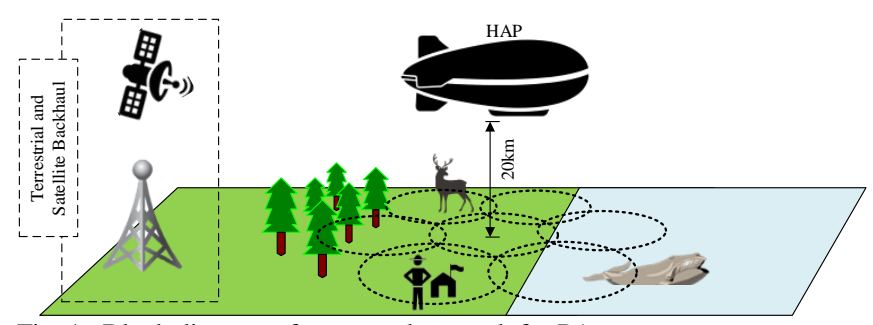

Fig. 1. Block diagram of suggested network for PAs. 


\section{A. HAPS Coverage}

We assume that each individual HAPS antenna provides coverage for a particular cell on the ground. We also assume that all cells have the identical coverage area (same size), which helps supporting a uniform device density throughout the HAPS coverage. Such uniform cell size can be achieved by properly adjusting the beam widths of the respective onboard antennas as will be shown later in the article.

For modeling purposes, hexagons are typically used to represent a cell and the tessellated hexagonal cells are organized in rings or tiers as shown in fig. 2(a). The number of cells within the $k^{\text {th }}$ tier equals to $6 \mathrm{k}$ and the cumulative number of cells up to the $k^{\text {th }}$ tier is given by:

$$
S_{k}=3 k^{2}+3 k+1 \quad k=0,1,2,3, \ldots
$$

The inner diameter, $d_{k}$, of a hexagon is calculated as $d_{k}=$ $\frac{\sqrt{3}}{2} r(2 k+1)$, where $\mathrm{r}$ represents the radius of a cell. Finally, the HAPS coverage, $R_{k}$, is obtained by (2) [19].

$$
R_{k}=r \sqrt{S_{k}} \quad k=0,1,2,3, \ldots
$$

\section{B. Device Density}

We assume that IoT devices operate with a very low duty cycling, i.e., the fraction of time an IoT device is ON. For example, a duty cycling of 0.01 means that each device only occupies a carrier $1 \%$ of the time, which would allow the carrier to serve 100 devices. Low duty cycling is a natural feature of IoT applications as the sensor devices basically transmit a small message once in few minutes or hours. The low duty cycling helps the HAPS system to connect a very large number of devices in each cell and hence support high device density (DD). For a HAPS with $N$ total number of carriers, $D D$ is thus derived as:

$$
D D=\frac{N}{\text { Duty Cycling } \times \text { Cell Area }}
$$

\section{Antenna Gain and Size}

If we approximate hexagons with a circle, we get the beamwidth of the antennas in each tier (refer to fig. 2(b)) as:

$$
=\left\{\begin{array}{cc}
\tan ^{-1}\left(\frac{r}{H}\right) & k=0 \\
\tan ^{-1}\left(\frac{d_{k}+0.134 r}{H}\right)-\tan ^{-1}\left(\frac{d_{k-1}-0.134 r}{H}\right) & k>0
\end{array}\right.
$$

Assuming that all antennas in a given tier have the same beamwidth, total antenna size, which affects the size, weight, and power $(\mathrm{SWaP})$ imposed by the antennas, can be expressed as:

$$
S_{\text {total }}=f\left(\Phi_{0}\right)+\sum_{i=1}^{k} 6 k f\left(\Phi_{i}\right),
$$

Where $f(\Phi)$ depends on the frequency and type of antenna. For example, we have $f(\Phi)=\pi\left(\frac{58 \lambda}{2 \Phi}\right)^{2}$ for dish antenna.

Fig. 2(c) shows a spherical coordinate system with the HAPS placed at its center and $\varphi$ is its azimuth angle. Fig. 2(d) describes antenna pattern based on $\varphi$. Equation (8) is a simple

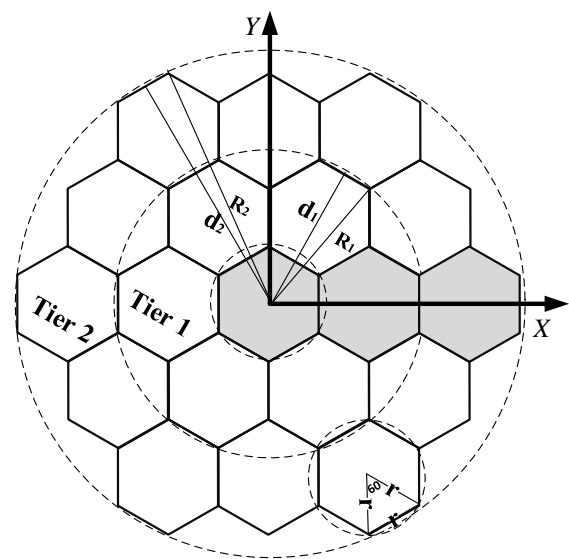

(a) A typical cellular pattern.

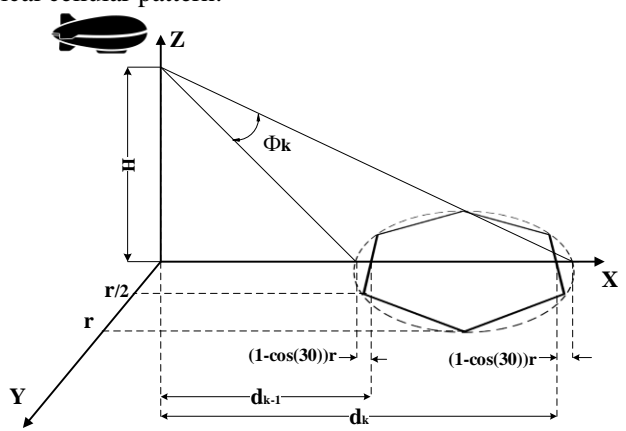

(b) Geometry of beam width.

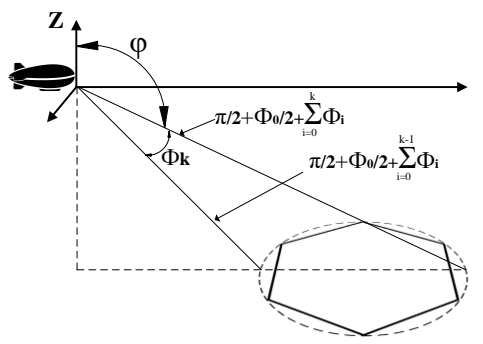

(c) Angle of beam width.

Fig. 2. Antenna gain and beamwidth model.

approximation for antenna gain where $\Phi_{k}$ is in degrees [4].

$$
G_{k}=\left\{\begin{array}{cc}
\frac{G_{0}}{\left(\Phi_{k}\right)^{2}} & \frac{\pi}{2}+\frac{\Phi_{0}}{2}+\sum_{i=1}^{k} \Phi_{i} \leq \phi \leq \frac{\pi}{2}+\frac{\Phi_{0}}{2}+\sum_{i=1}^{k-1} \Phi_{i} \\
0 & \text { otherwise, }
\end{array}\right.
$$

Where $G_{0}=29000$.

\section{Path Loss}

Path loss at a location $(\mathrm{x}, \mathrm{y})$ within the HAPS coverage comprises of air-to-ground ( $\mathrm{A} 2 \mathrm{G})$ absorbing loss and scattering (AaS) loss:

$$
L(x, y)=L_{A 2 G}(x, y)+L_{A a S}(x, y)
$$

Several models have been proposed for the A2G channels. We adopt the model proposed in [20], where $a, b, \eta_{L o S}$ and $\eta_{N L o S}$ are environment-dependent constants. 


$$
\begin{array}{r}
L_{A 2 G}(x, y)=\frac{A}{1+a \exp \left(-b\left[\tan ^{-1}\left(\frac{H}{\sqrt{x^{2}+y^{2}}}\right)-a\right]\right)} \\
+10 \log _{10}\left(H^{2}+x^{2}+y^{2}\right)+B
\end{array}
$$

$L_{A a S}$ is affected by the length of propagation path through fog, cloud, snow, or vegetation. For protected area coverage, we consider the propagation through the forest as the main source of scattering loss. Fig. 3 illustrates that the propagation length, $d(x, y)$, through the forest can be derived from the HAPS height and the height of the forest vegetation. For the forest model, we have [21]:

$$
\begin{gathered}
\left.L_{\text {Aas }}(x, y)=A_{m}\left(1-e^{\left(-d(x, y) \omega / A_{m}\right.}\right)\right) \\
\text { where: } A_{m}=A_{1} f^{v}, d(x, y)=\frac{h \sqrt{x^{2}+y^{2}+H^{2}}}{H}
\end{gathered}
$$

Where $d(x, y)$ is in meter, $f$ is the frequency in $\mathrm{MHz}$ and $A_{l}, \omega$ and $v$ are environment-dependent constants.

Clearly, path loss experienced at different locations will be different within a given cell. The average path loss within tier $\mathrm{k}$ can be obtained as follows (we use the gray cells in fig 2(a) for approximation):

$$
=\left\{\begin{array}{c}
\frac{4}{\sqrt{3}} \int_{0}^{d_{k}}\left(\int_{0}^{\frac{\sqrt{3}}{3} x+r} 10^{\left(\frac{L(x, y)}{10}\right)} d y\right) d x \quad k=0 \\
\frac{2}{\frac{3 \sqrt{3}}{2} r^{2}} \int_{d_{k-1}}^{d_{k}}\left(\int_{0}^{\frac{\sqrt{3}}{3} x-\frac{\sqrt{3}}{3} d_{k-1}+\frac{r}{2}} 10^{\left(\frac{L(x, y)}{10}\right)} d y+\int_{0}^{-\frac{\sqrt{3}}{3} x+\frac{\sqrt{3}}{3} d_{k}+\frac{r}{2}} 10^{\left(\frac{L(x, y)}{10}\right)} d y\right) d x \quad k>0 \\
\overline{L_{k}}=10 \log _{10}\left(\overline{l_{k}}\right)
\end{array}\right.
$$

\section{E. Coupling Loss}

Coupling loss at location $(\mathrm{x}, \mathrm{y}), C L(x, y)$, is defined as the net signal loss from the transmitter to the receiver after considering the path loss plus any other constant cabling loss at the transmitter minus the antenna gains:

$$
C L(\mathrm{x}, \mathrm{y})=L(x, y)+L_{\text {other }}-G_{t x}-G_{r x}
$$

Where $L_{o t h e r}$ is a constant cabling related loss, $G_{t x}$ and $G_{r x}$ are antenna gains at transmitter and receiver, respectively. Recall that antenna gains must be adjusted for different tiers to ensure uniform cell size across the tiers. Hence antenna gains are obtained as follows assuming that the ground devices use

\begin{tabular}{|c|c|c|c|c|}
\hline \multirow{2}{*}{ Specification } & \multirow{2}{*}{$\begin{array}{l}\text { Standard } \\
\text { Name }\end{array}$} & \multicolumn{3}{|c|}{ Coupling Loss } \\
\hline & & $144 \mathrm{~dB}$ & $154 \mathrm{~dB}$ & $164 \mathrm{~dB}$ \\
\hline \multirow{2}{*}{$\begin{array}{c}\text { Battery Life } \\
\text { (year) }\end{array}$} & LTE-m (Cat M1) & 22.3 & 8.7 & 0.9 \\
\hline & NB-IoT (standalone) & 20 & 7.9 & 1.4 \\
\hline \multirow{2}{*}{$\begin{array}{l}\text { Uplink data rate } \\
\text { (kbps) }\end{array}$} & LTE-m (Cat M1) & 40.1 & 3.1 & 0.167 \\
\hline & NB-IoT (standalone) & 18.8 & 2.6 & 0.343 \\
\hline \multirow{2}{*}{$\begin{array}{c}\text { Downlink data rate } \\
(\mathrm{kbps})\end{array}$} & LTE-m (Cat M1) & 76.6 & 9.9 & 0.8 \\
\hline & NB-IoT (standalone) & 19.1 & 8.7 & 1 \\
\hline \multirow{2}{*}{$\begin{array}{l}\text { Latency } \\
\text { (s) }\end{array}$} & LTE-m (Cat M1) & 0.2 & 0.6 & 8.5 \\
\hline & NB-IoT (standalone) & 0.3 & 0.7 & 5.1 \\
\hline
\end{tabular}
omnidirectional antennas $\left(G_{o d}\right)$ :

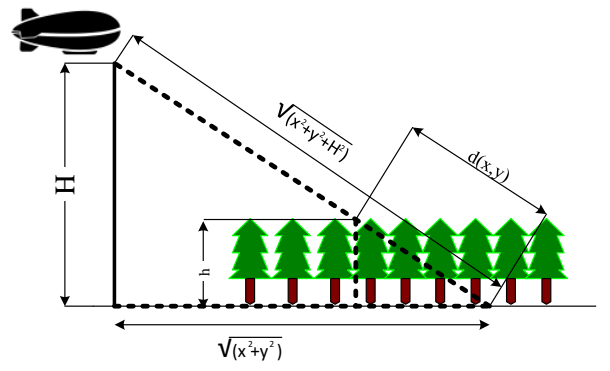

Fig. 3. Scattering loss through the forest. $H, h$, and $d(x, y)$ represent, respectively, the HAPS height, the height of the forest, and the propagation length through the forest vegetation.
TABLE I

EFFECT OF CL ON PERFORMANCE LTE-M AND NB-IOT

$$
\begin{aligned}
& G_{t x}=\left\{\begin{array}{lr}
G_{o d} & \text { in uplink time } \\
G_{k} & k=0,1,2, \ldots
\end{array}\right.
\end{aligned}
$$

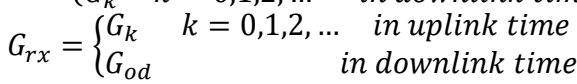

$\mathrm{CL}$ is an important factor that affects the battery life, data rates, and latency and hence has been chosen by 3GPP as the metric to evaluate coverage[14]. Table I shows how CL affects battery life, data rate, and lartency for LTE-m and NB-IoT. The battery life values in this table are calculated assuming that the uplink and downlink packet size is 200 and 65 bytes, respectively, reporting interval for the IOT devices is two hours, and the device is powered by two AA batteries. Latency values are based on messages with 85-byte length [14]

Given the important of CL, it is important to anaylze CL when designing a HAPS network. We derive expressions for both the average CL and the uniformity of the CL within the HAPS coverage. The average CL in a cell within the $k^{\text {th }}$ tier can be written as.

$$
\overline{C L_{k}}=\overline{L_{k}}+L_{\text {other }}-G_{t x}-G_{r x}
$$

The average of CL in the whole HAPS coverage area is then obtained as:

$$
\overline{C L}=10 \log _{10}\left(\frac{10^{\left(\frac{\overline{C L_{0}}}{10}\right)}+\sum_{j=1}^{k}\left(6 k \times 10^{\left(\frac{\overline{C L_{J}}}{10}\right)}\right)}{S_{k}}\right)
$$

To analyze the uniformity of CL within a cell, we propose to measure the difference between the maximum and minimum $\mathrm{CL}$ in that cell. The minimum and maximum CL of a cell occur at the location which is closest and farthest to the HAPS, respectively. They can be obtained for the $k^{\text {th }}$ cell as follows (we use gray color cells in fig. 2(a) for approximation)

$$
\begin{gathered}
C L_{\text {min }}{ }^{k}=\left\{\begin{array}{cc}
L(0,0)+L_{\text {other }}-G_{t x}-G_{r x} & k=0 \\
L\left(d_{k-1}, 0\right)+L_{\text {other }}-G_{t x}-G_{r x} & k>0
\end{array}\right. \\
C L_{\text {max }}{ }^{k}= \begin{cases}L\left(d_{1}, r\right)+L_{\text {other }}-G_{t x}-G_{r x} & k=0 \\
L\left(d_{k}, r\right)+L_{\text {other }}-G_{t x}-G_{r x} & k>0\end{cases}
\end{gathered}
$$

The uniformity of CL within a cell in tier $\mathrm{k}$ is thus obtained as (18). CL uniformity for the entire HAPS coverage is then given by (19):

$$
\Delta_{C L}{ }^{k}=C L_{\max }{ }^{k}-C L_{\min }{ }^{k}
$$




$$
\overline{\Delta_{C L}}=10 \log _{10}\left(\frac{10^{\left(\frac{\Delta C L^{0}}{10}\right)}+\sum_{j=1}^{k}\left(6 k \times 10^{\left(\frac{\Delta C L^{k}}{10}\right)}\right)}{S_{k}}\right)
$$

\section{NUMERICAL RESULTS AND DISCUSSION}

In this section, we conduct numerical experiments to study the relationships between various parameters of a HAPS-based cellular system.

\section{A. Spectrum requirements in HAPS cellular IoT systems}

In Table II, we review the various frequency ranges and bandwidth that are made available for LTE-m and NB-IoT [14]. We also show the number of carriers available for each frequency range by considering the fact that NB-IoT and LTE$\mathrm{m}$ use only $180 \mathrm{kHz}$ and $1.4 \mathrm{MHz}$ carriers, respectively. We can see that $1.7-2.1 \mathrm{GHz}$ band has large bandwidth, which provides large number of carriers: 2,388 for NB-IoT and 307 for LTE$\mathrm{m}$. However, the question is: how many carriers are needed to cover a certain protected area? We answer this question next.

Fig. 4 illustrates the number of carriers needed to meet different device density and duty cycle requirements of protected area monitoring. We can see that the number of required carriers increases exponentially with the increasing cell radius. This is because the number of devices to serve increases directly as a function of the cell area. Note that duty cycling has a small value because a node may send the only 100 bits every 12 hours.

The total number of carriers must be controlled for multiple reasons. First, there may not be enough carriers available for a suitable frequency band if cells are too large. Second, part of the area covered by a HAPS may also be covered by village or town, which necessitates sharing the same spectrum with

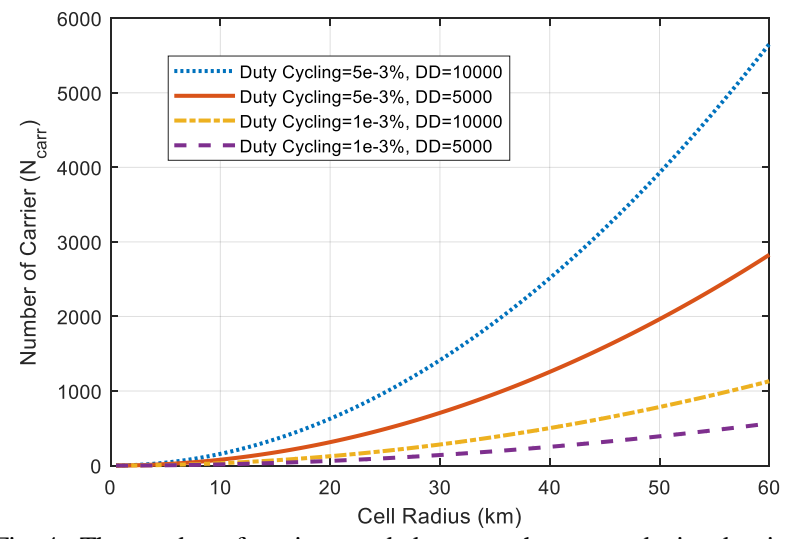

Fig. 4. The number of carriers needed to meet the proper device density.

TABLE II

SPECTRUM ALLOCATION TO LTE-M AND NB-IOT. THE LAST TWO COLUMNS SHOW THE NUMBER OF AVAILABLE CARRIERS.

\begin{tabular}{|c|c|c|c|}
\hline Frequency Range $(\mathrm{MHz})$ & Bandwidth & NB-IoT & LTE-M \\
\hline Around 450 & $5 \mathrm{MHz}$ & 27 & 3 \\
\hline $700-1000$ & $256 \mathrm{MHz}$ & 1422 & 182 \\
\hline Around 1500 & $35 \mathrm{MHz}$ & 194 & 25 \\
\hline $1700-2100$ & $430 \mathrm{MHz}$ & 2388 & 307 \\
\hline $2500-2700$ & $264 \mathrm{MHz}$ & not supported & 188 \\
\hline
\end{tabular}

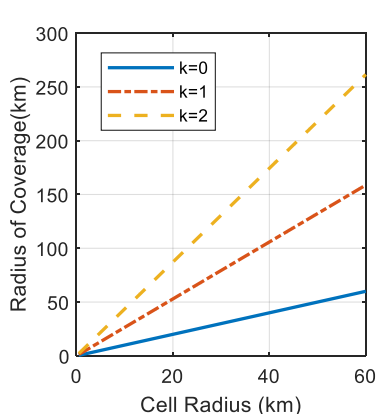

(a) The relation between the radius of cell and radius of coverage.

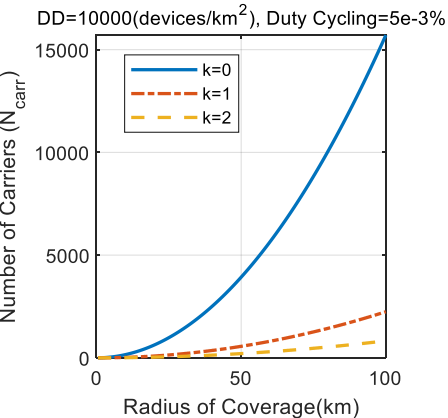
as a function of coverage radius. (b) The number of required carriers

Fig. 5. coverage radius vs. the radius of cell and number of required carriers for different tier cellular network topologies.

terrestrial infrastructure. Fig. 5(a) shows the relationship between the radius of cell and radius of coverage. We can see that, for the same coverage radius, cell radius can be reduced by deploying multiple tier $(\mathrm{k}>0)$ cellular networks. For example, to cover an area with a radius of $50 \mathrm{~km}$, the required cell radius is also $50 \mathrm{~km}$ for $\mathrm{k}=0$, while it is reduced to only $10 \mathrm{~km}$ if we configure the network with $\mathrm{k}=2$. The effect of $\mathrm{k}$ on the total number of required carriers is shown in fig. 5(b). We can see that with the 1.7-2.1GHz band (2,300 NB-IoT carriers), a large protected area of radius $90 \mathrm{~km}$ can be easily monitored with NBIoT for multi-tier cellular systems $(\mathrm{k}>0)$, but the coverage has to be limited to less than $35 \mathrm{~km}$ of radius if the coverage is provided using a non-tiered cellular configuration $(\mathrm{k}=0)$.
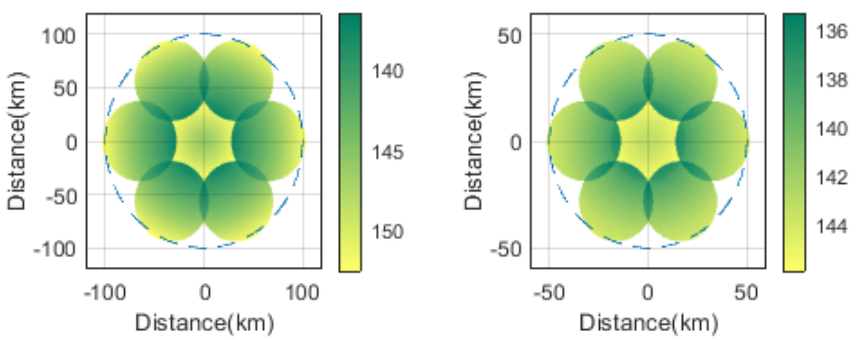

(a) For $\mathrm{k}=1, \mathrm{R}_{\mathrm{k}}=100 \mathrm{~km}$ without forest cover.

(b) For $\mathrm{k}=1, \mathrm{R}_{\mathrm{k}}=50 \mathrm{~km}$
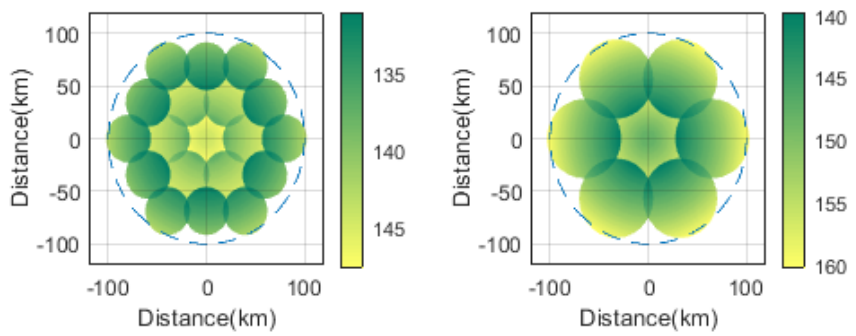

(c) for $\mathrm{k}=2, \mathrm{R}_{\mathrm{k}}=100 \mathrm{~km}$ without forest cover.

(d) For $\mathrm{k}=1, \mathrm{R}_{\mathrm{k}}=100 \mathrm{~km}$ with forest cover.

Fig. 6. CL distribution, Values from [20] are used for environment-dependent constants in the suburbs. The f, $v$, and $\omega$ are $2 \mathrm{GHz}, 1.37$, and 0.42 , respectively [21]. Other signal loss and leakage are assumed to be $35 \mathrm{~dB}$ and the IoT devices are assumed to be equipped with omnidirectional antennas. 


\section{B. Coupling loss performance in HAPS cellular networks}

To visualize how CL changes over the cell area, we have plotted CL distributon in Fig. 6. We can see how CL will decrease with a lower radius of coverage (fig. 6(a) vs. 6(b)) or a higher k (fig. 6(a) vs. 6(c)), as well as the effect of forest cover on it (fig. 6(a) vs. 6(d)).

Fig. 7 illustrates the average CL and the uniformity of CL for suburban areas with and without forest cover. As expected, CL increases with forest, but we also make the following interesting observations:

1. Both average $C L$ and uniformity of $C L$ increases with coverage radius irrespective of $\mathrm{k}$. This is because larger coverage increases path loss for the boundary regions and larger cells sizes cause more variations for path loss with in the cell. CL and CL uniformity therefore put constraints on HAPS coverage radius.

2. For the same HAPS coverage radius, both average CL and uniformity of CL decrease with an increasing $\mathrm{k}$. This is because, for the same coverage, smaller cells are required for a larger $\mathrm{k}$. Note that smaller cells are equivalent to more antenna gain and more signal loss compensation. This means that, from the CL perspective, it is better to organize the HAP network into as many tiers as possible.

\section{Antenna size}

Total antenna size as a function of coverage radius for different cellular topologies is shown in Fig. 8(a). We can make

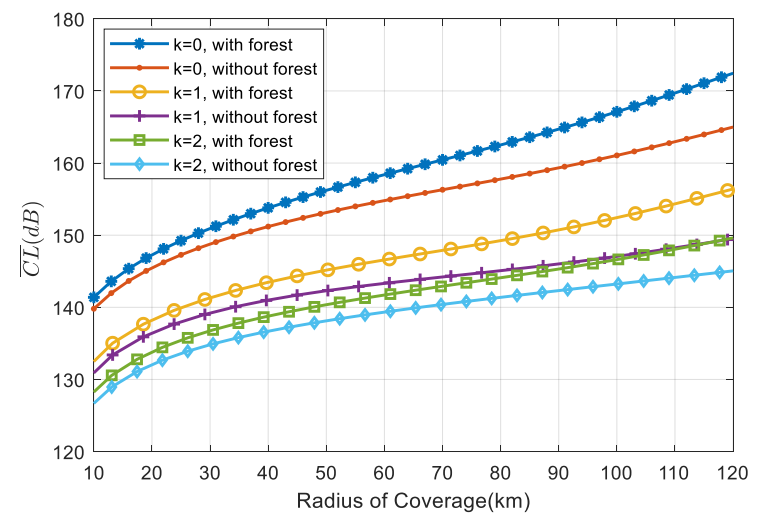

(a) Average CL

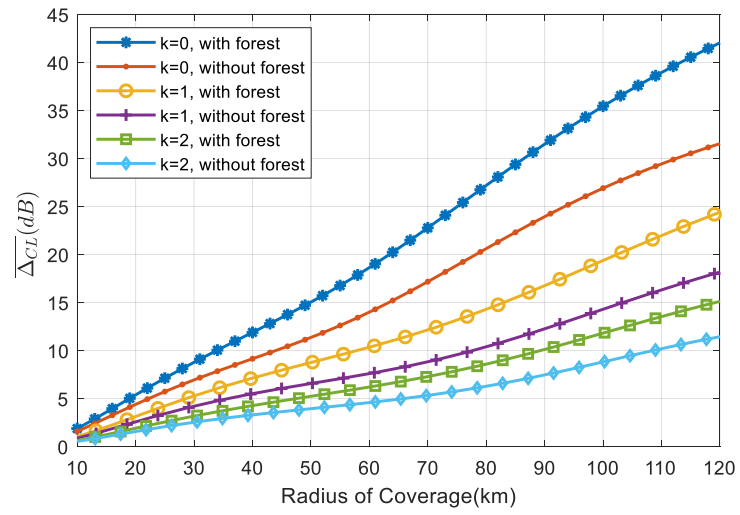

(b) Uniformity of CL

Fig. 7. Impact of $k$ and coverage radius $(\mathrm{R})$ on the average $\mathrm{CL}$ and uniformity of $\mathrm{CL}$ for suburban areas with and without forest. Simulation conditions are the same as in Fig. (6).

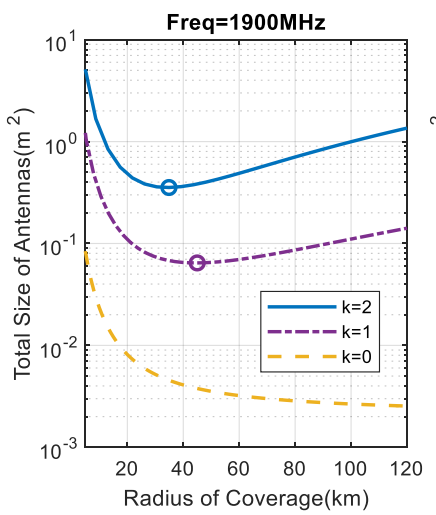

(a) Different tier.

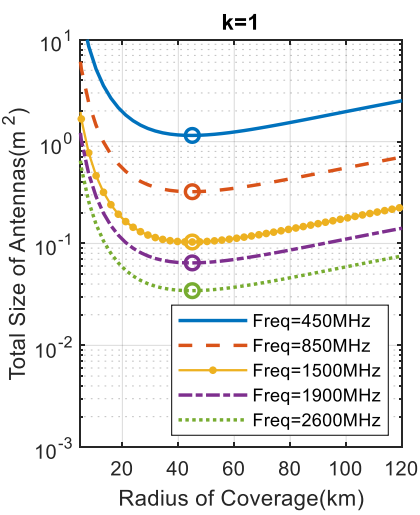

(b) Different frequencies
Fig. 8. Total antenna size vs. coverage radius.

the following observations:

1. The antenna size monotonically decreases with an increasing coverage radius for $\mathrm{k}=0$. This is because the beam width (beam angle) also increases monotonically for the cell as the coverage radius increases. Note that the higher the beam width, the lower the antenna gain, hence the lower the antenna size as analyzed earlier in Section III.C.

2. There is an optimum coverage radius that minimizes the total antenna size when a multi-tier $(\mathrm{k}>0)$ cellular topology is used. This is because the antenna size for the cell in $\mathrm{k}=0$ decreases but the antenna size for cells in $\mathrm{k}>0$ increases as these cells are pushed further away from the center when the center cell increases.

3. Fig. 8(b) shows that the coverage radius that minimizes the total antenna size does not depend on the frequency of communication. This can be explained by the fact that although the coverage radius affects the antenna beam width, the frequency does not affect the beam width as modeled in (4).

\section{Maximizing HAPS coverage under CL and antenna size constraints}

For a given $k$, the HAPS coverage can be increased by increasing the cell size. However, as seen in the previous subsection, increasing cell size directly increases CL, uniformity of CL, and antenna size, all of which would have practical constraints. In this section, we analyze the HAPS coverage maximization under these constraints by solving (20) for different $k$.

$$
\begin{array}{cc}
\text { maximize } \\
\text { subject to } \\
\\
& \overline{C L_{k}} \leq \alpha \\
\overline{\Delta_{C L}} \leq \beta \\
S_{\text {total }} \leq \gamma \\
r \leq \delta
\end{array}
$$

Fig. 9(a) shows the effect of parameter $\alpha$ on the maximum $\left(R_{k}\right)$ for a given set of $\beta, \gamma$, and $\delta$. We see that the HAPS radius can be increased by tolerating more coupling loss up to a certain point, after which $R_{k}$ is basically limited by the other constraints and hence it cannot be increased any further. The saturation point in $R_{k}$ is higher for a higher $k$ and it is reached quicker with 
a smaller $\alpha$. For example, for the parameter set of Fig. 8(a), we can achieve HAPS coverage up to $98 \mathrm{~km}$ by tolerating a CL of only $145 \mathrm{~dB}$ if $\mathrm{k}=2$. But for $\mathrm{k}=0$, the coverage is limited to only $35 \mathrm{~km}$ while having to tolerate a higher CL of $153 \mathrm{~dB}$. This shows, that organizing the HAPS coverage into a higher-tier network can help to achieve a higher coverage range.

Fig. 9(b) illustrates the impact of $\alpha$ and $\beta$ on maximizing $R_{k}$. We can see that the HAPS radius can be increased by tolerating more coupling loss up to a certain point, after which $R_{k}$ saturates and is limited by the other constraints. The saturation point of $R_{k}$ is higher for a higher $\beta$ but we have to tolerate higher CL to reach there. For example, for the parameter set of Fig. 8(b), we can achieve a HAPS coverage up to $106 \mathrm{~km}$ for $\beta=14 \mathrm{~dB}$ by

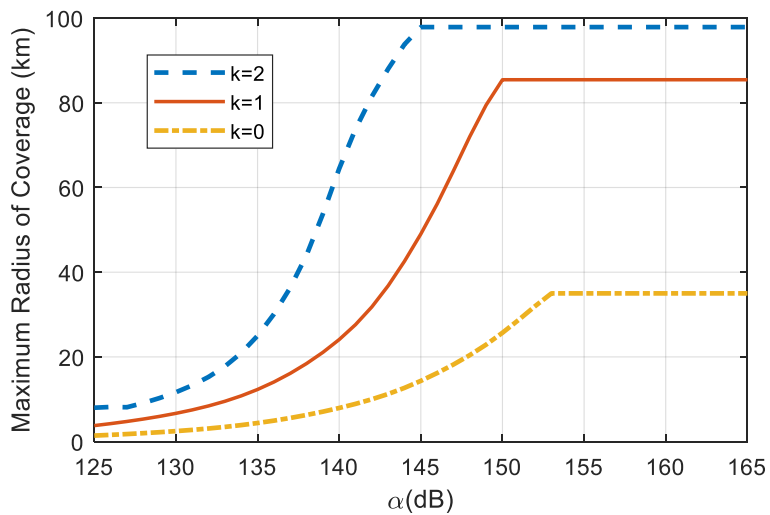

(a) Impact of $\alpha$ and $\mathrm{k}\left(\delta=35 \mathrm{~km}, \gamma=10 \mathrm{~m}^{2}\right.$, and $\beta=15 \mathrm{~dB}$.)

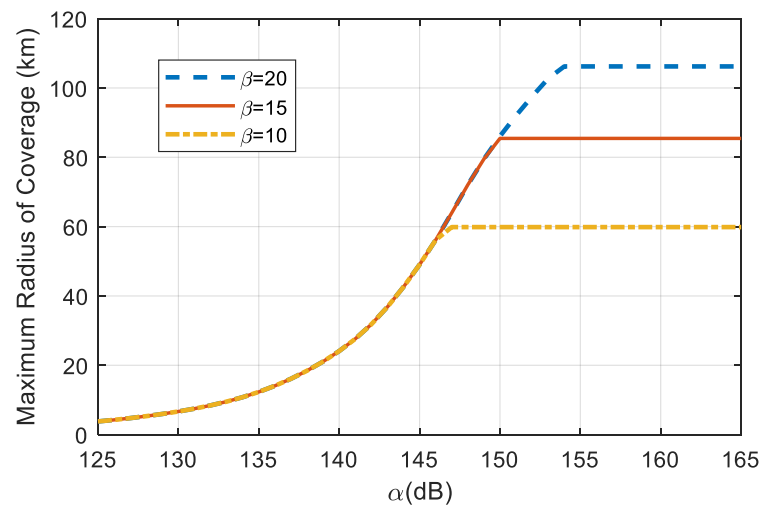

(b) Impact of $\alpha$ and $\beta\left(\delta=35 \mathrm{~km}, \gamma=4 \mathrm{~m}^{2}\right.$, and $\mathrm{k}=1$.)

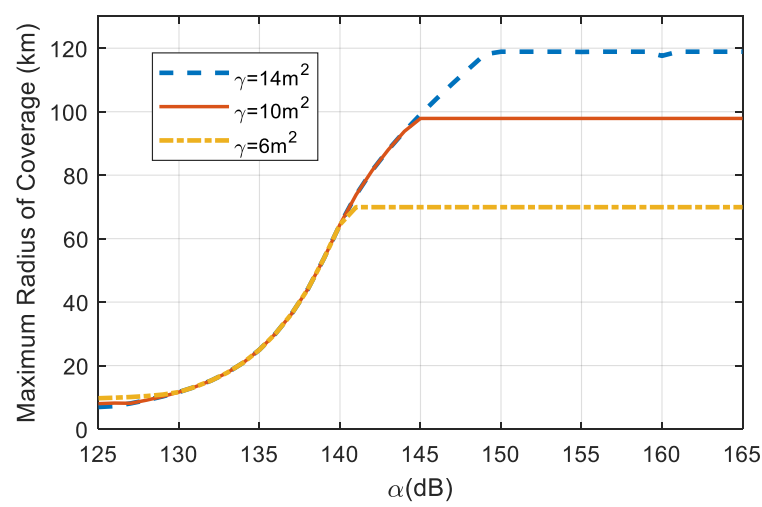

(c) Impact of $\alpha$ and $\gamma(\delta=35 \mathrm{~km}, \mathrm{k}=2$, and $\beta=15 \mathrm{~dB}$.)

Fig. 9. The effect of different constraints on maximum $R_{k}$. tolerating a CL of $147 \mathrm{~dB}$. However, for $\beta=10$, the HAPS coverage can be increased only up to $59 \mathrm{~km}$.

Finally, Fig. 9(c) shows the impact of $\alpha$ and $\gamma$. We can see that the HAPS radius can be increased by tolerating more coupling loss up to a certain point, after which $R_{k}$ is limited by the other constraints. A larger $\gamma$ allows a larger coverage.

\section{CONCLUSION}

We have modeled HAPS cellular networks to support IoT networks in large protected areas. We have derived closed-form expressions to study the impact of practical constraints such as device density requirements, minimum coupling loss targets, and antenna size limitations on maximizing the HAPS coverage. Our study shows that a large HAPS coverage of more than $100 \mathrm{~km}$ radius can be achieved to support high-density NBIoT and LTE-m IoT services with good quality of service and acceptable antenna size.

\section{REFERENCES}

[1] "Global Coverage | Protected Planet Digital Report." https://livereport.protectedplanet.net/chapter-2 (accessed Feb. 12, 2020).

[2] E. D. Ayele, K. Das, N. Meratnia, and P. J. M. Havinga, "Leveraging BLE and LoRa in IoT network for wildlife monitoring system (WMS)," in 2018 IEEE 4th World Forum on Internet of Things (WF-IoT), 2018, pp. $342-348$

[3] G. Hu et al., "Self-powered 5G NB-IoT system for remote monitoring applications," Nano Energy, vol. 87, p. 106140, Sep. 2021, doi: 10.1016/J.NANOEN.2021.106140.

[4] M. Mozaffari, W. Saad, M. Bennis, and M. Debbah, "Efficient Deployment of Multiple Unmanned Aerial Vehicles for Optimal Wireless Coverage," IEEE Commun. Lett., vol. 20, no. 8, pp. 1647-1650, Aug. 2016, doi: 10.1109/LCOMM.2016.2578312.

[5] A. Fotouhi, M. Ding, and M. Hassan, "Flying Drone Base Stations for Macro Hotspots," IEEE Access, vol. 6, pp. 19530-19539, 2018, doi: 10.1109/ACCESS.2018.2817799.

[6] A. Fotouhi, H. Qiang, M. Ding, M. Hassan, L. G. Giordano, A. GarciaRodriguez, J. Yuan, "Survey on UAV Cellular Communications: Practical Aspects, Standardization Advancements, Regulation, and Security Challenges," IEEE Commun. Surv. Tutorials, vol. 21, no. 4, pp. 3417-3442, 2019, doi: 10.1109/COMST.2019.2906228.

[7] "Zephyr - U UAV - Airbus." https://www.airbus.com/defence/uav/zephyr.html\#introduction (accessed Aug. 13, 2021)

[8] "Loon - X, the moonshot factory." https://x.company/projects/loon/ (accessed Aug. 12, 2021).

[9] "SERVICE https://www.hapsmobile.com/en/service/ (accessed Aug. 12, 2021)

[10] Y. Yao, Y. Sun, C. Phillips, and Y. Cao, "Movement-Aware Relay Selection for Delay-Tolerant Information Dissemination in Wildlife Tracking and Monitoring Applications," IEEE Internet Things J., vol. 5, no. 4, pp. 3079-3090, Aug. 2018, doi: 10.1109/JIOT.2018.2831439.

[11] J. M. Nassar et al., "Compliant lightweight non-invasive standalone 'Marine Skin' tagging system," npj Flex. Electron., vol. 2, no. 1, p. 13, Dec. 2018, doi: 10.1038/s41528-018-0025-1.

[12] T. O. Olasupo, "Wireless Communication Modeling for the Deployment of Tiny IoT Devices in Rocky and Mountainous Environments," IEEE Sensors Lett., vol. 3, no. 7, pp. 1-4, 2019, doi: 10.1109/lsens.2019.2918331.

[13] N. Ahmed, D. De, and I. Hussain, "Internet of Things (IoT) for Smart Precision Agriculture and Farming in Rural Areas," IEEE Internet Things J., vol. 5, no. 6, pp. 4890-4899, Dec. 2018, doi: 10.1109/JIOT.2018.2879579.

[14] J. Bergman, O. Liberg, M. Sundberg, E. Wang, J. Bergman, and J. Sachs, Cellular Internet of Things: Technologies, Standards, and Performance. Academic Press, 2017.

[15] O. M. Bushnaq, A. Chaaban, and T. Y. Al-Naffouri, "The Role of UAVIoT Networks in Future Wildfire Detection," IEEE Internet Things J., pp. 1-1, 2021, doi: 10.1109/JIOT.2021.3077593. 
[16] M. Alzenad, A. El-Keyi, F. Lagum, and H. Yanikomeroglu, "3-D Placement of an Unmanned Aerial Vehicle Base Station (UAV-BS) for Energy-Efficient Maximal Coverage," IEEE Wirel. Commun. Lett., vol. 6, no. 4, pp. 434-437, Aug. 2017, doi: 10.1109/LWC.2017.2700840.

[17] Y. Shibata, N. Kanazawa, M. Konishi, K. Hoshino, Y. Ohta, and A. Nagate, "System Design of Gigabit HAPS Mobile Communications," IEEE Access, vol. 8, pp. 157995-158007, 2020, doi: 10.1109/ACCESS.2020.3019820.

[18] Y. Shibata, N. Kanazawa, K. Hoshino, Y. Ohta, and A. Nagate, "A Study on Cell Configuration for HAPS Mobile Communications," in 2019 IEEE 89th Vehicular Technology Conference (VTC2019-Spring), Apr. 2019, vol. 2019-April, pp. 1-6, doi: 10.1109/VTCSpring.2019.8746491.

[19] M. Prvan, J. Ožegović, and A. B. Mišura, "On Calculating the Packing Efficiency for Embedding Hexagonal and Dodecagonal Sensors in a Circular Container," Math. Probl. Eng., vol. 2019, pp. 1-16, Jul. 2019, doi: $10.1155 / 2019 / 9624751$.

[20] A. Al-Hourani, S. Kandeepan, and S. Lardner, "Optimal LAP Altitude for Maximum Coverage," IEEE Wirel. Commun. Lett., vol. 3, no. 6, pp. 569-572, Dec. 2014, doi: 10.1109/LWC.2014.2342736.

[21] "Attenuation in vegetation P Series Radiowave propagation ITU-R P.833-9," $2016 . \quad$ [Online]. Available: https://www.itu.int/dms_pubrec/itu-r/rec/p/R-REC-P.833-9-201609I!!PDF-E.pdf.

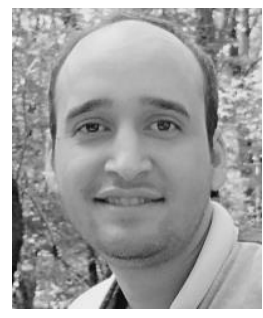

Masoud Ardiny received the M.Sc. degree in telecommunication engineering from Shahed University, Tehran, Iran, in 2013. He is a senior researcher in some laboratories and startup companies in Iran. His research interests include satellite communications, satellite imaging, modern imaging methods, drone base stations for cellular, internet of things, and embedded systems.

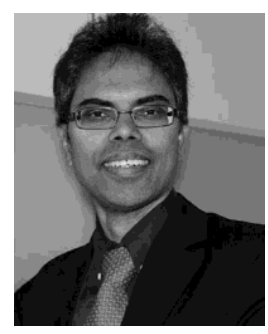

Mahbub Hassan (Senior Member, IEEE) received the M.Sc. degree in computer science from the University of Victoria, Canada, and the Ph.D. degree in computer science from Monash University, Australia. He is a Full Professor with the School of Computer Science and Engineering, University of New South Wales, Sydney, Australia. He served as an IEEE Distinguished Lecturer and held visiting appointments at universities in USA, France, Japan, and Taiwan. He has coauthored three books, over 200 scientific articles, and a U.S. patent. He served as an Editor or a Guest Editor for many journals, including IEEE Communications Magazine, IEEE Network, and IEEE Transactions on Multimedia. His current research interests include mobile computing and sensing, nanoscale communication, and wireless communication networks.

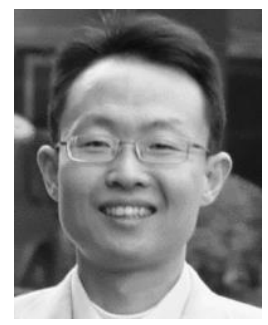

Ming Ding (Senior Member, IEEE) received the B.S. and M.S. degrees (with first class Hons.) in electronics engineering and the Doctor of Philosophy (Ph.D.) degree in signal and information processing from Shanghai Jiao Tong University (SJTU), Shanghai, China, in 2004, 2007, and 2011, respectively. From April 2007 to September 2014, he was a Researcher/Senior Researcher/Principal Researcher with the Sharp Laboratories of China, Shanghai, China. He was also the Algorithm Design
Director and Programming Director for a system-level simulator of future telecommunication networks with the Sharp Laboratories of China for more than 7 years. He is currently a Senior Research Scientist with Data61, CSIRO, Sydney, NSW, Australia. He has authored more than 100 papers in IEEE journals and conferences, all in recognized venues, and about 20 3GPP standardization contributions, and also a Springer book Multi-point Cooperative Communication Systems: Theory and Applications. He also holds 16 U.S. patents and coinvented another more than 100 patents on $4 \mathrm{G}$ or $5 \mathrm{G}$ technologies in CN, JP, and EU. He is the Editor of the IEEE Transactions on Wireless Communications. Besides, he is or has been a Guest Editor or Co-Chair or Co-Tutor or TPC member of several IEEE top-tier journals or conferences, such as, the IEEE Journal on Selected Areas in Communications, the IEEE Communications Magazine, and the IEEE Globecom Workshops. He was the lead speaker of the industrial presentation on unmanned aerial vehicles in IEEE Globecom 2017, which was awarded as the Most Attended Industry Program in the conference. Also, he was awarded in 2017 as the Exemplary Reviewer for the IEEE Transactions on Wireless Communications.

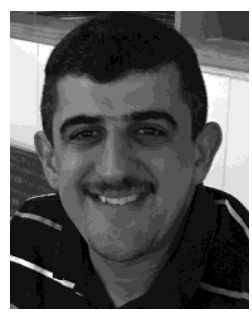

Omar Salim (Member, IEEE) received the B.Sc. and M.Sc. degrees in electronics and communication engineering from Mosul University, Mosul, Iraq, in 2001 and 2004, respectively, and the Ph.D. degree in signal processing for communication engineering from USQ University, Toowoomba, QLD, Australia, in 2015.,He was a Post-Doctoral Research Fellow with the School of Electrical and Mechanical, USQ University, from 2015 to 2016. He joined Monash University, Clayton, VIC, Australia, as a Research Assistance and a Teaching Associate, in 2017. He is currently a PostDoctoral Research Fellow with the Department of Electrical and Computer Systems Engineering, Monash University. He has authored several high-quality conference and journal articles. His research interests include signal processing for communication and RFID-based sensor systems and digital signal processing implementation for 3-D video communication systems and RFID reader-based chipless and chipped RFID tag and sensors.

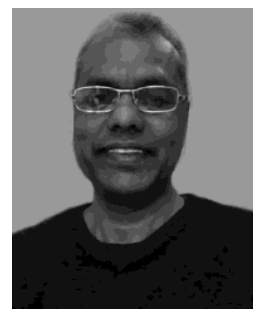

Nemai Karmakar (Senior Member, IEEE) received the M.Sc. degree in electrical engineering from the University of Saskatchewan, Saskatoon, SK, Canada, in 1991, and the Ph.D. degree from the University of Queensland, Brisbane, QLD, Australia, in 1999.,He is currently an Associate Professor with the Department of Electrical and Computer Systems Engineering, Monash University, Melbourne, VIC, Australia. He has authored or coauthored over 220 refereed journal and conference papers, 24 book chapters, and eight books. He holds nine international patent applications on chipless RFID. He has over 20 years of teaching, design, and development experience in antennas, microwave active and passive circuits, and RFIDs in Canada, Australia, and Singapore. 